\title{
Carbon C 11 Glutamine
}

National Cancer Institute

\section{Source}

National Cancer Institute. Carbon C 11 Glutamine. NCI Thesaurus. Code C137987.

Upon intravenous administration of 11C-glutamine, glutamine is preferentially taken up by cancer cells. Upon PET, the biodistribution and uptake by cancer cells can be assessed. Tumor cells use the amino acid glutamine for nutritional purposes including energy production and growth; as tumor cells proliferate more rapidly than normal healthy cells, glutamine uptake is higher in certain cancer cells. 